\title{
Clinical Experience with Intravenous Lipid Emulsion for Drug-Induced Cardiovascular Collapse
}

\author{
Ann-Jeannette Geib • Erica Liebelt • Alex F. Manini • \\ On behalf of the Toxicology Investigators' Consortium \\ (ToxIC)
}

Published online: 12 October 2011

(C) American College of Medical Toxicology 2011

\begin{abstract}
Intravenous lipid emulsion (ILE) is an emerging therapy for refractory cardiotoxicity due to lipid-soluble drugs. The purpose of this study was to assess survival to hospital discharge, effects on hemodynamic parameters, and adverse event occurrence for patients who were treated with ILE as part of the resuscitative effort for drug-induced cardiotoxicity. This is a multicenter retrospective chart review of inpatients at three tertiary referral medical centers receiving ILE for drug-induced cardiotoxicity between November 2007 and March 2009. Nine cases with drug-induced cardiovascular collapse, defined as cardiac arrest or refractory shock, were selected for review if patients received either bolus or infusion of ILE in any combination. No interventions were done. The main outcome measures were survival to hospital discharge, effect on hemodynamic parameters, and adverse event. Hemodynamic vital signs (heart rate, systolic blood pressure, diastolic blood pressure, calculated mean arterial pressure [MAP]) were measured before administration of ILE and up to five measurements (if available) were recorded after administration of ILE. Attribution of adverse events was determined by
\end{abstract}

A.-J. Geib $(\bowtie)$

Medical Toxicology Program,

Department of Emergency Medicine,

Robert Wood Johnson Medical School,

New Brunswick, NJ 08901, USA

e-mail: ajgeib@hotmail.com

E. Liebelt

Department of Pediatrics,

University of Alabama Birmingham School of Medicine,

Birmingham, AL, USA

\section{A. F. Manini}

Division of Medical Toxicology,

Department of Emergency Medicine,

Mount Sinai School of Medicine,

New York, NY, USA assignment of Naranjo adverse drug reaction (ADR) likelihood score (3) with adjudication of three medical toxicologists; disagreements were settled by majority consensus. Of nine cases identified based on inclusion criteria (three cardiac arrest, six refractory shock), five (55\%) survived to hospital discharge. ILE regimens were bolus alone in five patients and bolus plus infusion in four patients. Hemodynamic trends in response to ILE demonstrated no difference in MAP immediately pre- and post-administration of ILE $(p=\mathrm{NS})$. Administration of infusion (versus boluses alone) did not demonstrate a statistically significant improvement in MAP. Adverse events due to ILE therapy that were categorized as "possible" or "probable" based on Naranjo scores included lipemia, digit amputation, lung injury, renal failure, and deep venous thrombosis. ILE administered to patients with drug-induced cardiovascular collapse was associated with $55 \%$ survival but with clinically significant adverse effects. At this time, ILE should be restricted to cardiotoxicity involving cardiac arrest or refractory shock until further prospective studies can better evaluate risks and benefits of ILE therapy.

Keywords Lipid therapy · Poisoning · Cardiac arrest

\section{Introduction}

Intravenous lipid emulsion (ILE) therapy is an emerging, but unproven therapy for refractory cardiotoxicity due to lipid-soluble drugs [1]. Its promise was first recognized in a rat model of bupivacaine toxicity [2] and has gained visibility in the clinical arena after reports of its use for bupivacaine and other drug toxicities [3].

Evidence for ILE in the management of drug-related cardiotoxicity is limited at this time to animal studies and 
human case reports. Animal models have demonstrated improved survival from drug-induced cardiotoxicity from bupivacaine, clomipramine, propranolol, atenolol, and verapamil when ILE is administered during resuscitative efforts. Human case reports in the literature document recovery from cardiovascular collapse due to poisoning from bupivacaine, ropivacaine, bupropion/lamotrigine, mepivicaine/bupivacaine, and levobupivacaine. [4]

To date, no human case series or observational studies report the use of ILE to treat cardiotoxicity from drugs other than local anesthetics. To evaluate the potential risks and benefits of ILE for this indication, we report a multicenter study drawing upon data from the Toxicology Investigators' Consortium (ToxIC) focusing on the application of ILE therapy to cardiotoxicity from drugs other than local anesthetics. The objective was to assess survival to hospital discharge, effects on hemodynamic parameters, and adverse event occurrence for patients who were treated with ILE as part of the resuscitative effort for drug-induced cardiotoxicity.

\section{Methods}

Study Setting and Design Cases were identified through the Toxicology Investigators' Consortium (ToxIC), a multicenter registry of more than 45 medical toxicology practices throughout the USA. ToxIC is an effort sponsored by the American College of Medical Toxicology. This chart review was performed in a retrospective fashion using electronic medical record review of databases from November 2007 to March 2009.

Definitions All patients in the study presented with a history of acute drug toxicity defined as clinical symptoms or injury caused by exposure to prescription pharmaceuticals. Prior to receiving ILE, cardiovascular collapse was defined as one or both of either cardiac arrest (pulselessness requiring chest compressions) or refractory hypotension (hypotension requiring $>1$ pressor). Survival in this study was defined as survival to hospital discharge regardless of neurological status at time of discharge. Ventilator-dependent respiratory failure (VDRF) was defined as prolonged ventilation requirement $>7$ days. Adverse events were defined as a presenting or acquired condition that led to prolongation of hospitalization or need for medical or surgical intervention. Attribution of adverse events was determined by assignment of the Naranjo adverse drug reaction (ADR) likelihood [5].

Data Collection Contributors submitted cases using a uniform data collection instrument. Data were entered into an Excel ${ }^{\mathrm{TM}}$ spreadsheet and later into SPSS for statistical analysis. Investigators obtained IRB approval from their institutions. In-hospital adverse events from chart review were abstracted by one reviewer (AJG).

Measurements Hemodynamic vital signs (heart rate, systolic blood pressure, diastolic blood pressure, calculated mean arterial pressure $[\mathrm{MAP}]$ ) were measured before administration of ILE and up to five measurements (if available) were recorded after administration of ILE.

The Naranjo ADR likelihood was used in this study and has been described elsewhere [5]. Briefly, adjudicators independently answered ten questions related to whether IVLE caused the ADR assigning answers of "yes", "no", or "don't know" with points assigned for each answer (see Table 1). ADRs were classified as follows: $\geq 9$, definite; 5 8 , probable; $1-4$, possible; $\leq 0$, doubtful.

Statistical Analysis Descriptive statistics are reported as percentages or means with standard error. Continuous and categorical variables were analyzed using the student's $t$ test and Fisher's exact test, respectively. All $p$ values were twotailed with $5 \%$ alpha. Computer analysis was performed using SPSS v. 17 software (SPSS Inc., Chicago, IL).

\section{Results}

Characteristics of Patients Over the study period we identified nine patients $(77 \%$ female, age range 18 60 years) who received ILE therapy for cardiovascular drug toxicity. Patient characteristics are summarized in Table 2.

Drug Exposures Multi-drug overdose occurred in 66\% (ranging from two to four drugs). Drug class exposures included antidepressants $(n=7)$, antihypertensives $(n=7)$, anticonvulsants $(n=1)$, and muscle relaxants $(n=1)$. Drug exposures are summarized in Table 1.

Lipid Emulsion Regimens All patients received ILE in a 20\% (20 gm/dL) concentration of lipid emulsion, however dosing was inconsistent. Two thirds of patients received bolus dosing only (two single, three double, two triple), while the others received bolus followed by an infusion. One patient received two rounds of bolus plus infusion. Lipid emulsion regimens for each case are summarized in Table 2. Neither administration of multiple boluses (compared with one bolus, $p=\mathrm{NS}$ ) nor an infusion (compared with no infusion, $p=\mathrm{NS}$ ) were associated with improved survival.

Hemodynamic Response to ILE Hemodynamic trends in response to ILE demonstrated no difference in MAP immediately pre- and post-administration of ILE $(p=\mathrm{NS})$. 
Table 1 Adverse drug reactions (ADR) scoring system (adapted from Naranjo et al. [5])

\begin{tabular}{|c|c|c|c|}
\hline ADR query & Yes & No & Don't know \\
\hline Are there previous conclusive reports on this reaction? & +1 & 0 & 0 \\
\hline Did the adverse event appear after the suspected drug was administered? & +2 & -1 & 0 \\
\hline Did the adverse reaction improve when the drug was discontinued or a specific antagonist was administered? & +1 & 0 & 0 \\
\hline Did the adverse reaction reappear when the drug was readministered? & +2 & -1 & 0 \\
\hline Are there alternative causes (other than the drug) that could on their own have caused the reaction? & -1 & +2 & 0 \\
\hline Did the reaction reappear when a placebo was given? & -1 & +1 & 0 \\
\hline Was the drug detected in the blood (or other fluids) in concentrations known to be toxic? & +1 & 0 & 0 \\
\hline Was the reaction more severe when the dose was increased or less severe when the dose was decreased? & +1 & 0 & 0 \\
\hline Did the patient have a similar reaction to the same or similar drugs in any previous exposure? & +1 & 0 & 0 \\
\hline Was the adverse event confirmed by any objective evidence? & +1 & 0 & 0 \\
\hline
\end{tabular}

ADRs were classified as follows: $\geq 9$, definite; $5-8$, probable; $1-4$, possible; $\leq 0$ doubtful

Administration of an infusion (versus boluses alone) did not demonstrate a statistically significant improvement in MAP (see Fig. 1).

Survival Survival to hospital discharge occurred in five $(55 \%)$ subjects, of whom $80 \%$ were neurologically intact. Of the three patients with cardiac arrest prior to ILE, two $(66.7 \%)$ survived. Care was withdrawn by the health care proxy in two out of four deaths.

ILE-Related Adverse Events In the five survivors, the most common adverse events were lipemia $(n=3)$, DVT $(n=3)$, acute lung injury $(n=3)$, and acute renal failure $(n=3)$. Neurological impairment in study subjects included delirium, short-term memory loss, and anoxic brain injury. Adverse events and likelihood of attribution to ILE based on Naranjo scores are summarized in Table 3.

\section{Discussion}

Intravenous lipid emulsion (ILE) administration was associated with 55\% survival. However, administration of an infusion (versus boluses alone) did not demonstrate a statistically significant improvement in MAP (Fig. 1). Clinically significant adverse events which were "possibly" or "probably" related to ILE use in survivors included: lipemia (60\%), DVT (60\%), acute lung injury (60\%), and acute renal failure (60\%).
Table 2 Characteristics of patients treated with ILE for cardiovascular drug toxicity
$C A$ cardiac arrest, $F$ female, ILE intravenous lipid emulsion, $G t t$ infusion, $M$ male

\begin{tabular}{|c|c|c|c|c|}
\hline Case no. (age, sex) & Indication & Drug exposure & ILE regimen & Survival \\
\hline \multirow[t]{2}{*}{1 (28 years, $M)$} & \multirow[t]{2}{*}{ Shock } & $\begin{array}{l}\text { Duloxetine } \\
\text { Lamotrigine }\end{array}$ & \multirow[t]{2}{*}{ Bolus $(\times 2)$} & \multirow[t]{2}{*}{ No } \\
\hline & & Verapamil & & \\
\hline $2(34$ years, F) & $\mathrm{CA}$ & Amlodipine & Bolus $(\times 3)$ & No \\
\hline $3(18$ years, F) & Shock & $\begin{array}{l}\text { Amlodipine } \\
\text { Lisinopril }\end{array}$ & Bolus $(\times 2)$ & Yes \\
\hline $4(33$ years, F) & $\begin{array}{l}\text { Shock } \\
\text { Post-CA }\end{array}$ & Amitriptyline & Bolus $(\times 1)$ & Yes \\
\hline $5(60$ years, $F)$ & Shock & $\begin{array}{l}\text { Amoxapine } \\
\text { Verapamil }\end{array}$ & Bolus $(\times 2)+\mathrm{Gtt}$ & Yes \\
\hline $6(30$ years, F) & $\begin{array}{l}\text { Shock } \\
\text { CA }\end{array}$ & $\begin{array}{l}\text { Amitriptyline } \\
\text { Cyclobenzaprine }\end{array}$ & Bolus $(\times 3)$ & Yes \\
\hline 7 (44 years, F) & Shock & $\begin{array}{l}\text { Amitriptyline } \\
\text { Verapamil }\end{array}$ & Bolus $+\mathrm{Gtt}$ & No \\
\hline $8(60$ years, $M)$ & Shock & Bupropion & Bolus $+\mathrm{Gtt}$ & No \\
\hline \multirow[t]{3}{*}{$9(56$ years, $F)$} & \multirow[t]{3}{*}{ Shock } & $\begin{array}{l}\text { Amitriptyline } \\
\text { Citalopram }\end{array}$ & \multirow[t]{3}{*}{ Bolus $(\times 1)$} & \multirow[t]{3}{*}{ Yes } \\
\hline & & Metoprolol & & \\
\hline & & Nifedipine & & \\
\hline
\end{tabular}




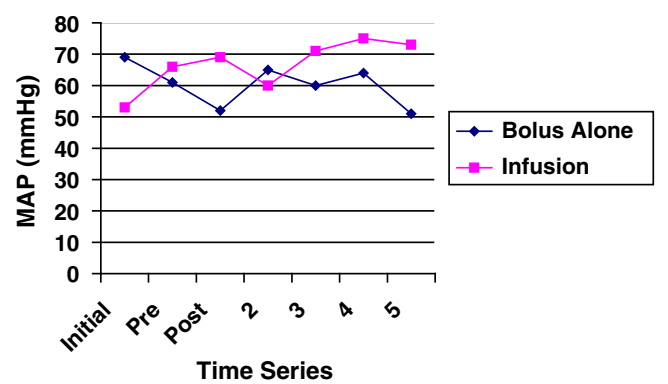

Fig. 1 Time series of mean MAP comparing cases of ILE bolus alone versus bolus plus infusion. Each subsequent number across the time series represents chronological order. Due to the retrospective nature of the study, there was no standardization with regards to intervals of time that each subsequent time-point was measured. ILE intravenous lipid emulsion, $M A P$ mean arterial pressure, $\mathrm{mmHg}$ millimeters of mercury

With 55\% survival in this series of patients with otherwise non-survivable cardiotoxic insult, ILE is likely to have facilitated survival for these patients. ILE is currently hypothesized to ameliorate drug cardiotoxicity by three mechanisms: provision of a "lipid sink" by providing a separate pharmacologic compartment into which the lipophilic drug may diffuse [6]; delivery of substrate to an energydepleted myocardium [7]; and improving function of select ion channels (e.g., calcium and sodium) present on the cell membrane in order to overcome myocardial conduction delays and poor inotropy [6]. Animal models of drug cardiotoxicity suggest that ILE administration may have superior survival efficacy over classic resuscitative therapies such as epinephrine $[1,8]$ and vasopressin [1].

Patients who survived in our study may have benefited from metabolic effects of ILE. Metabolic acidosis can exacerbate myocardial depression in toxicity from many drug classes. The ensuing shock state causes myocardial energy consumption to rely on glucose rather than free fatty acids [9]. The myocardium's limited glycogen stores are quickly depleted. ILE may shift myocardial metabolism back to dependence on free fatty acids [9], which may in turn improve inotropy and chronotropy.

Concerns have been raised about the safety of ILE in critically ill patients with regards to risk of acute lung injury (ALI). The theoretical mechanisms of pulmonary toxicity due to ILE are severalfold and include the following: local hypoperfusion, injury from hypoxic pulmonary vasocon-
Table 3 Adverse events and likelihood of attribution to ILE

\begin{tabular}{|c|c|c|c|}
\hline Case no. & Doubtful & Possible & Probable \\
\hline 1 & Death $[-2]$ & & Lipemia [7] \\
\hline 2 & Death $[-3]$ & & \\
\hline 3 & & Acute lung injury [1] & \\
\hline \multirow[t]{4}{*}{4} & & $\begin{array}{l}\text { Acute lung injury [1] } \\
\text { Acute renal failure [1] }\end{array}$ & \\
\hline & & Diabetic ketoacidosis [2] & \\
\hline & & Anoxic brain injury [2] & \\
\hline & & VDRF [2] & \\
\hline \multirow[t]{9}{*}{5} & & Acute lung injury [1] & Lipemia [7] \\
\hline & & Acute renal failure [1] & \\
\hline & & DVT (lower extremity) [1] & \\
\hline & & Toe amputations $\times 8[1]$ & \\
\hline & & Atrial fibrillation [1] & \\
\hline & & Deconditioning [1] & \\
\hline & & Decubitus ulcer [1] & \\
\hline & & Sepsis [1] & \\
\hline & & VDRF [2] & \\
\hline \multirow[t]{2}{*}{6} & & DVT (upper extremity) [1] & \\
\hline & & $\begin{array}{l}\text { Short-term memory loss [1] } \\
\text { Aspiration [1] }\end{array}$ & \\
\hline \multirow[t]{5}{*}{7} & Death $[-2]$ & $\mathrm{DIC}[1]$ & Lipemia [7] \\
\hline & & DVT (upper extremity) [1] & \\
\hline & & Mesenteric infarction [1] & \\
\hline & & Metabolic acidosis [1] & \\
\hline & & Severe hyperthermia [1] & \\
\hline 8 & Death $[-2]$ & Delirium [1] & \\
\hline 9 & & Hypoglycemia [1] & \\
\hline
\end{tabular}

Naranjo score in [brackets]

DIC disseminated intravascular coagulation, $D V T$ deep venous thrombosis, ILE intravenous lipid emulsion, $V D R F$ ventilator-dependent respiratory failure 
striction, and effects from resultant localized edema. Oleic acid, which comprises about $22 \%$ of the free fatty acids in ILE preparations, is associated with the development of ALI in animal models [10]. Human studies using ILE as its labeled indication for nutritional use describe a low incidence of ALI but the causative role of triglycerides has recently been called into question [11]. In our population, ALI resulted in $33 \%$ of patients' post-administration of ILE. While our study cannot prove causality, the Naranjo ADR likelihood [5] was classified as a score of $1 / 12$ (i.e., possibly related). Additionally, it is difficult to calculate risk of ALI in our subset of patients who were already predisposed for ALI due to hypotension, drug effect on alveolar capillary permeability, or aspiration. Future studies should address the risks of ILE raised in this dataset, including those of ALI.

Three patients had lipemia reported on laboratory analysis which may cause either inaccuracies or delayed result reporting. Physicians using ILE should be aware of the potential for laboratory inaccuracies caused by lipemia $[12,13]$. However, long-term effects of ILE on survival or adverse events are not known and should be taken into consideration. A longitudinal study could provide valuable information to this end.

Limitations Our study is subject to several limitations including all those inherent to its retrospective nature. Conclusions may also be limited by lack of uniform application (including degree of shock and pharmacology of the drug toxicity), other antidotes (e.g., bicarbonate), and lack of a control group or prognostic information about each drug's toxicity. In addition, the reliance on clinical history to determine the drugs taken in overdose and small sample size prohibits more sophisticated analysis. Using mean MAP values in our subjects, we estimate a sample size of at least 100 patients would be required to have adequate power to detect a significant difference in hemodynamic parameters with a $t$ test.

\section{Conclusions}

In this study, there was over $50 \%$ survival for patients receiving ILE as part of the resuscitation for drug-induced cardiovascular collapse. Clinically significant adverse effects were common and possibly associated with ILE administration. At this time, ILE administration is reasonable for the treatment of refractory cardiovascular collapse due to exposures to lipid-soluble drugs. However, use should be restricted to cardiac arrest or refractory shock until further prospective study can better evaluate risks and benefits of ILE therapy.

Acknowledgments We wish to thank J. Ward Donovan, MD, FACEP, FACMT, and Tracey Helmick, RN, CCRN, for their assistance in making this study possible.

\section{References}

1. Jamaty C, Bailey B, Larocque et al (2010) Lipid emulsions in the treatment of acute poisoning: a systematic review of human and animal studies. Clin Tox 48:1-27

2. Weinberg GL, VadeBoncouer T, Ramaraju GA, Garcia-Amaro MF, Cwik MJ (1998) Pretreatment or resuscitation with a lipid infusion shifts the dose-response to bupivacaine-induced asystole in rats. Anesthesiology 88(4):1071-1075

3. Dalgleish D, Kathawaroo S (2005) Lipid emulsion to treat bupivacaine toxicity. Anaesthesia 60:817-830

4. Lipidrescue.org (2007) LipidRescue resuscitation in the medical literature. www.lipidrescue.org. Accessed 12 September 2011.

5. Naranjo CA, Busto U, Sellers EM et al (1981) A method for estimating the probability of adverse drug reactions. Clin Pharmacol Ther 30(2):239-245

6. Cave G, Harvey M (2009) Intravenous lipid emulsion as antidote beyond local anesthetic toxicity: a systematic review. Academic Emerg Med 16:815-824

7. Nelson RH, Prasad A, Lerman A et al (2007) Myocardial uptake of circulating triglycerides in nondiabetic patients with heart disease. Diabetes 56(2):527-530

8. Hiller DB, DiGregorio G, Ripper R et al (2009) Epinephrine impairs lipid resuscitation from bupivacaine overdose: a threshold effect. Anesthesiology 111(3):498-505

9. Ménard SL, Ci X, Frisch F et al (2009) Mechanism of reduced myocardial glucose utilization during acute hypertriglyceridemia in rats. Mol Imaging Biol 11(1):6-14

10. Ishitsuka Y, Moriuchi H, Yang C (2009) Effects of bolus injection of soybean-based fat emulsion and fatty acids on pulmonary gas exchange function. Biol Pharm Bull 32(3):500-503

11. Lekka M, Liokatis S, Nathanail C et al (2004) The impact of intravenous fat emulsion administration in acute lung injury. Am $\mathrm{J}$ Respir Crit Care Med 169:638-640

12. Randall AG, Garcia-Webb P, Beilby JP (1990) Interference by haemolysis, icterus and lipaemia in assays on the Beckman Synchron CX5 and methods for correction. Ann Clin Biochem 27:345-352

13. Meany D, Schowinsky J, Clarke W (2008) Effects of haemolysis and lipaemia on the COBAS salicylate and acetaminophen assays compared to GDS assays. Clin Biochem 41:1486-1488 